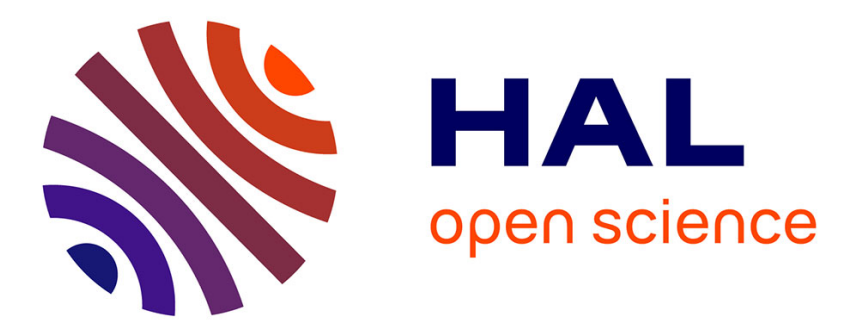

\title{
Generation of Excess Noise by Jets with Highly Disturbed Laminar Boundary-Layer Profiles
}

Christophe Bogey

\section{To cite this version:}

Christophe Bogey. Generation of Excess Noise by Jets with Highly Disturbed Laminar BoundaryLayer Profiles. AIAA Journal, 2021, 59, pp.569 - 579. 10.2514/1.j059610 . hal-03124046

\author{
HAL Id: hal-03124046 \\ https://hal.science/hal-03124046
}

Submitted on 9 Feb 2021

HAL is a multi-disciplinary open access archive for the deposit and dissemination of scientific research documents, whether they are published or not. The documents may come from teaching and research institutions in France or abroad, or from public or private research centers.
L'archive ouverte pluridisciplinaire HAL, est destinée au dépôt et à la diffusion de documents scientifiques de niveau recherche, publiés ou non, émanant des établissements d'enseignement et de recherche français ou étrangers, des laboratoires publics ou privés. 


\title{
Generation of excess noise by jets with highly disturbed laminar boundary-layer profiles
}

\author{
Christophe Bogey* \\ Univ Lyon, Ecole Centrale de Lyon, INSA Lyon, Université Claude Bernard Lyon I, CNRS, \\ Laboratoire de Mécanique des Fluides et d'Acoustique, UMR 5509, F-69134, Ecully, France
}

The generation of noise by jets with highly-disturbed laminar boundary-layer profiles at the nozzle exit, also referred to as initially nominally laminar jets in the literature, is investigated using large-eddy simulation and linear stability analysis. Four jets at a Mach number of 0.9 and a Reynolds number of $5 \times \mathbf{1 0}^{4}$, one with a non-laminar boundary-layer profile and three others with laminar profiles, are considered for exit peak turbulence intensities equal to $6 \%$ in the non-laminar case and to $9 \%$ in the laminar ones. The jets with laminar boundary-layer profiles all radiate greater sound pressure levels than the jet with a non-laminar profile but weaker initial disturbances. This particularly appears at high frequencies for the jets with a thinner boundary layer compared to the non-laminar case. These results are shown to be related to the dependence on the shape of the boundary-layer profile of the most unstable frequencies downstream of the nozzle. For a laminar profile, these frequencies are similar to those obtained downstream in the mixing-layer profiles, whereas they are higher for a non-laminar profile. Despite larger nozzle-exit flow disturbances, this leads to longer-term persistence of coherent large-scale structures in the shear layers, hence stronger velocity fluctuations and noise levels, for the present initially nominally laminar jets than for the other one.

\section{Nomenclature}

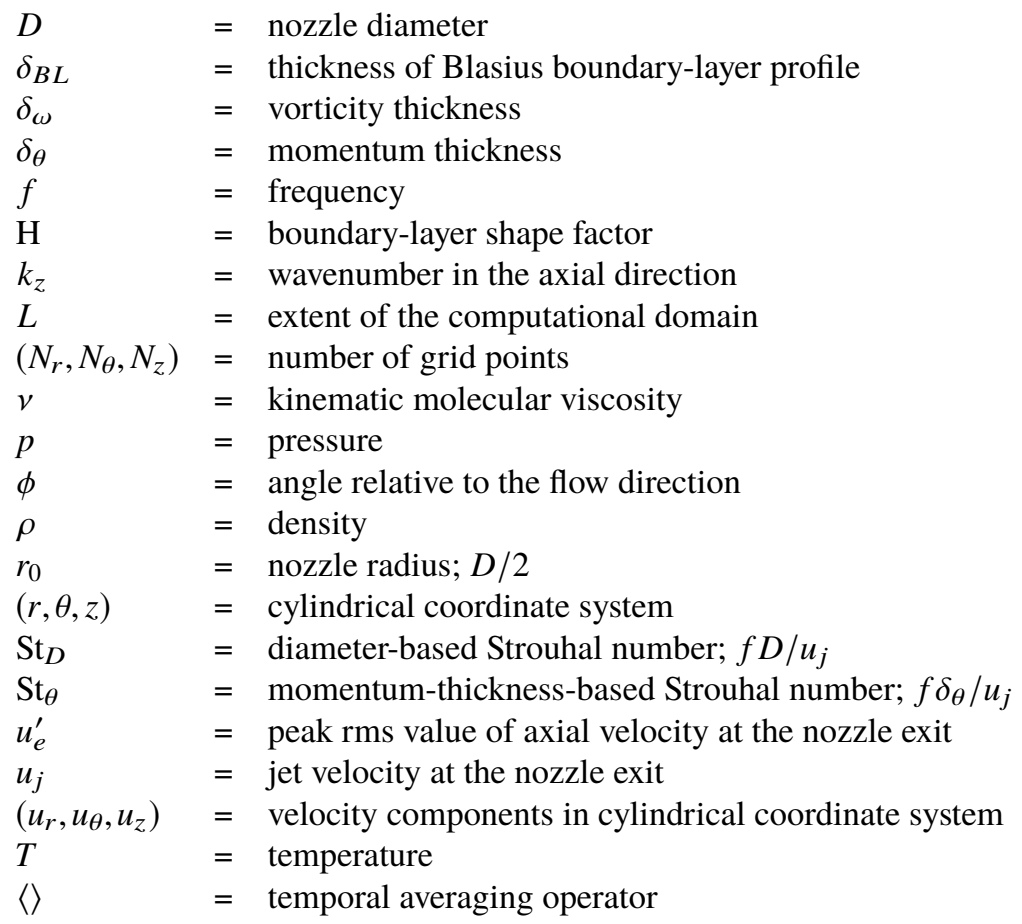

\section{Superscripts}

*CNRS Research Scientist, christophe.bogey@ec-lyon.fr, AIAA Senior Member \& Associate Fellow. 


$$
\text { , }=\text { fluctuation }
$$

Subscripts

$a$

$=$ ambient conditions

\section{Introduction}

The sensitivity of the noise of subsonic jets to the state of the nozzle-exit boundary layer has been recognized for at least fifty years. In 1964, for instance, Mollo-Christensen et al. [1] pointed out that, unlike initially turbulent jets, initially fully laminar jets may emit sound at a single frequency. For such conditions, usually encountered [2] for diameter-based Reynolds numbers $\operatorname{Re}_{D} \leq 10^{5}$, the velocity profile of the boundary layer agrees with the Blasius laminar profile and the turbulence intensities are lower than $1 \%$ at the nozzle exit. Therefore, linear instability waves initially grow, at frequencies corresponding to $S t_{\theta}=0.009-0.018$ according to experimental data [3-5], where $S t_{\theta}$ is the Strouhal number based on the exit boundary-layer momentum thickness. Farther downstream, they saturate and form vortex rings in the mixing layers, which subsequently merge and then degenerate into three-dimensional turbulence. This laminar-turbulent transition process leads to very large velocity fluctuations early on in the mixing layers, as reported by Bradshaw [6] in 1966, and to strong components in the acoustic far-field spectra, centered around half the instability wave peak frequency. This vortex-pairing noise can be suppressed by tripping the boundary layer inside the nozzle, as was done for example in the experiments of Zaman [2] and Bridges \& Hussain [7] and in the numerical simulations of Bogey et al. [8], so as to impose highly disturbed flow conditions at the exit.

However, highly disturbed nozzle-exit conditions do not necessarily imply fully turbulent boundary layers. On the contrary, several transitional conditions can be obtained between the fully laminar and the fully turbulent cases, typically for laboratory-scale jets at Reynolds numbers between $\operatorname{Re}_{D}=10^{5}$ and $5 \times 10^{5}$. One peculiar state for the boundary layer is the nominally laminar state [2], for which the velocity profile does not differ much from the Blasius profile, while the turbulence intensities are significant and can reach values as high as around $10 \%$. Such conditions have been measured in quite a few experiments [2, 9, 10] over the last years. More recently, their impact on noise generation has been discussed in studies dealing with the effects of the nozzle internal geometry on the acoustic fields of subsonic jets [11-14]. Initially nominally laminar jets were found to generate sound levels higher by 2-3 dB than initially turbulent jets, especially at high frequencies. In particular, it was observed in a series of experiments [11-13] for jets from the ASME and the conical nozzles, as illustrated by the far-field spectra of figure 1 At the exhaust of these nozzles, the boundary layer keeps a laminar shape up to Reynolds numbers around $6 \times 10^{6}$ in the ASME case, but is thicker and turbulent in the conical case according to Zaman [12]. The peak turbulence intensities also vary between $9 \%$ and 12\% with the ASME nozzle, but only between $6 \%$ and $8 \%$ with the conical nozzle. Therefore, the initially most disturbed jets emit more noise, which is rather unexpected and difficult to understand.

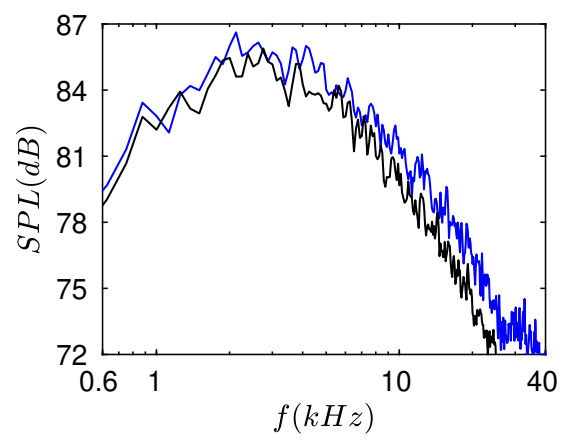

Fig. 1 Far-field sound pressure levels obtained at $\phi=60^{\circ}$ for Mach 0.896 jets using the conical and ASME nozzles [15].

These results motivated researchers to conduct additional experimental work on the subject. Fontaine et al. [14] considered initially highly disturbed jets with laminar and turbulent boundary-layer profiles. For three jets from short, medium and long nozzles of identical diameter, they measured higher levels in the acoustic field and larger velocity fluctuations a few diameters downstream of the nozzle for the jet with a laminar profile than for the two others with 
turbulent profiles. They also noticed a pronounced influence of the initial shear-layer thickness on the high-frequency noise components. Zaman [15] examined jets from six two-inch diameter nozzles, including the ASME and conical nozzles, and confirmed that a nominally laminar nozzle-exit boundary layer results in stronger noise components compared to a turbulent boundary layer, particularly on the high-frequency side of the sound spectra. He attributed this to the apparent presence and interactions of more organized vortical structures in the jet shear layers in the laminar case than in the turbulent one.

Numerical simulations have also been performed. Brès et al. [16] calculated two subsonic jets at a Reynolds number of $\operatorname{Re}_{D}=10^{6}$ with laminar and turbulent nozzle-exit boundary layers. They suggested that the greater high-frequency noise obtained in the laminar case is related to the difference in growth rate of the Kelvin-Helmholtz instability waves in the near-nozzle region. More recently, Bogey \& Sabatini [17] computed three Mach number 0.9 jets at a Reynolds number of $5 \times 10^{4}$ with an initial turbulence intensity of $6 \%$ and different boundary layers of momentum thickness of $2.8 \%$ of the nozzle radius. For the two jets with non-laminar exit profiles, the mixing layers contain weaker fluctuations, and the noise levels are lower by $2-3 \mathrm{~dB}$ compared to the jet with the laminar profile. These trends are illustrated in figure 2 showing the rms values of axial velocity fluctuations along the nozzle-lip line and pressure spectra obtained at $150 r_{0}$ from the nozzle for two jets with laminar and non-laminar exit profiles, referred to as Lam6\% and Turb6\% here (jetBL and jetT2 in [17]). They are explained by the difference in frequency of the initial instability waves. For a non-laminar boundary-layer profile, these waves develop at frequencies higher than the most unstable frequencies obtained after the boundary-layer/mixing-layer changeover. Therefore, they are rapidly damped, yielding weaker coherent large-scale structures in the shear layers compared to the laminar boundary-layer profile, which is consistent with the flow visualizations of Zaman [15].
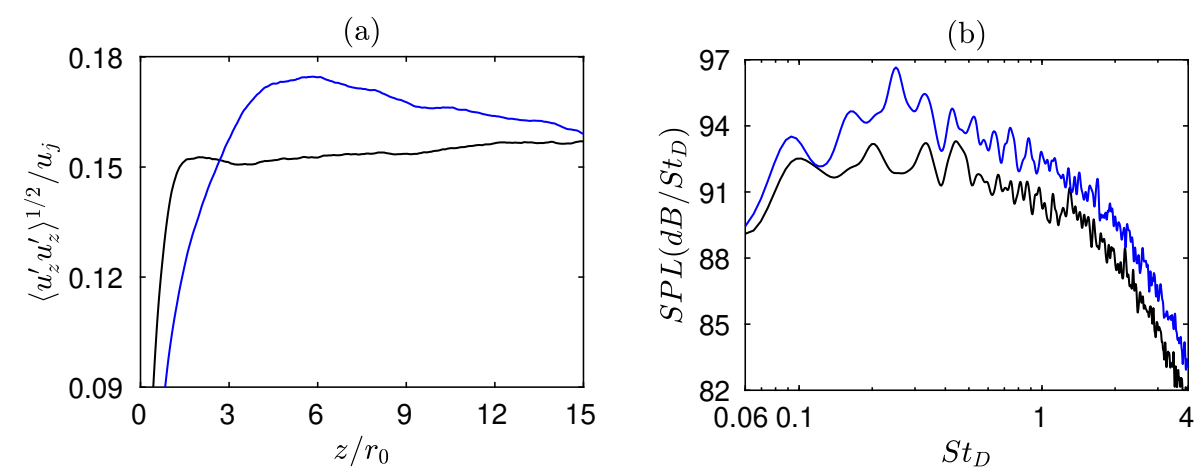

Fig. 2 Representation of (a) the rms values of axial velocity at $r=r_{0}$ and (b) far-field sound pressure levels for $\phi=9^{\circ}:-$ Turb6\%, — Lam6\% [17].

In this paper, the generation of excess noise by nominally laminar subsonic jets is investigated by comparing the flow and sound fields of Turb6\% with a non-laminar boundary-layer profile, with those of three initially highly disturbed jets with laminar boundary-layer profiles, computed using large-eddy simulations (LES). In the latter three jets, the nozzle-exit turbulence intensities are higher and the boundary-layer momentum thicknesses are similar to or smaller than those in Turb6\%, in order to roughly mimic the conditions measured just downstream of the ASME and conical nozzles. Therefore, the first objective will be to check whether, despite their larger initial disturbances, the initially nominally laminar jets radiate more noise and contain more coherent large-scale structures than the jet with a non-laminar boundary layer, in the same way as in the experiments. This is not obvious at first sight, and was not achieved in a previous numerical study [18] for two jets with experimental-like nozzle-exit boundary-layers. The origin of the excess noise and its sensitivity to the laminar boundary-layer thickness, in particular at high frequencies, will be discussed. A linear stability analysis will also be performed from the LES mean flow fields in order to reveal the growth rates and frequencies of the instability waves just downstream of the nozzle and their variations in the axial direction. The consequences on the presence and persistence of coherent large-scale structures in the mixing layers will be highlighted. It is worth noting that the present set of nominally laminar jets with various boundary-layer thicknesses will allow us to distinguish between the effects due to the higher-shear portion of the nozzle-exit profiles and those due to the form of the velocity profile alone. This was not possible in the work [17] mentioned above, in which the jet boundary layers have similar momentum thicknesses but various vorticity thicknesses, that is, different maximum velocity gradients. With the present jets, the different and sometimes opposite effects of the exit turbulence level, boundary-layer thickness and shape could also be examined, contrary to the previous study. 
The paper is organized as follows. The main parameters of the jets and numerical simulations are documented in section II. The jet flow and acoustic fields and the linear stability analysis are presented in section III. Concluding remarks are given in section IV. Finally, results obtained for jets with untripped boundary layers are shown in an appendix in order to further illustrate the effects of the nozzle-exit velocity profile.

\section{Parameters}

\section{A. Jet initial conditions}

Four isothermal round jets at a Mach number $\mathrm{M}=u_{j} / c_{a}=0.9$ and a Reynolds number $\operatorname{Re}_{D}=u_{j} D / v=5 \times 10^{4}$ are considered, where $u_{j}, D, c_{a}$ and $v$ are the jet velocity and diameter, the speed of sound in the ambient medium and the kinematic molecular viscosity. They originate at $z=0$ from a straight pipe nozzle of radius $r_{0}$ and length $2 r_{0}$, into a medium at rest of temperature $T_{a}=293 \mathrm{~K}$ and pressure $p_{a}=10^{5} \mathrm{~Pa}$. At the pipe inlet, at $z=-2 r_{0}$, a boundary-layer profile is imposed for the axial velocity, radial and azimuthal velocities are set to zero, pressure is equal to $p_{a}$ and temperature is determined by a Crocco-Busemann relation. In the pipe, the boundary layers are artificially tripped by adding random low-level vortical disturbances uncorrelated in the azimuthal direction at $z=-0.95 r_{0}$ [19], using a procedure generating velocity spectra at the nozzle exit in good agreement with spectra in wall-bounded flows [20]. The disturbance magnitudes are adjusted in order to achieve the desired level of peak turbulence intensity $u_{e}^{\prime} / u_{j}$ at the pipe exit, where $u_{e}^{\prime}$ is the maximum rms value of axial velocity fluctuations.

The four jets are denoted as Turb6\%, Lam9\%, Lam9\%thinner1 and Lam9\%thinner2 in what follows. Their upstream conditions were defined in previous studies [17, 21, 22]. For Turb6\%, the pipe-inlet velocity profile is a transitional boundary-layer profile with a momentum thickness and a shape factor, calculated without including density, of $\delta_{\theta}=0.029 r_{0}$ and $\mathrm{H}=1.52$, see in reference [17] for more details. The jet is forced so as to reach $u_{e}^{\prime} / u_{j}=6 \%$ at the nozzle exit. For the three other jets, the pipe-inlet profiles are Blasius laminar boundary-layer profiles of thickness $\delta_{B L}=0.25 r_{0}, 0.15 r_{0}$ and $0.09 r_{0}$, yielding $\delta_{\theta}=0.029 r_{0}, 0.017 r_{0}$ and $0.010 r_{0}$, for Lam9\%, Lam9\%thinner1 and Lam9\%thinner2, respectively. The boundary-layer forcing is tuned in order to obtain $u_{e}^{\prime} / u_{j}=9 \%$.

The profiles of mean and rms axial velocities calculated at the nozzle exit are presented in figure 3 . Their main properties are collected in table 1 In figure 3 a), the mean velocity profiles differ significantly, and have shape factors $\mathrm{H}=1.71$ for Turb6\% and between $\mathrm{H}=2.13$ and 2.24 for the three other jets. The shape factors indicate that the boundary-layer profiles are non-laminar (but not fully turbulent) in the first case and (nearly fully) laminar in the second one. From $z=-2 r_{0}$ up to $z=0$, they slighly increase for Turb6\% and decrease otherwise. Because of the the flow forcing inside the nozzle, the states of the exit boundary layers are more transitional than the laminar and turbulent states associated with the velocity profiles imposed at the inlet. The momentum thicknesses of the exit mean velocity profiles are equal to $\delta_{\theta}=0.0274 r_{0}$ for Turb6\%, and decrease from $\delta_{\theta}=0.0313 r_{0}$ down to $0.0116 r_{0}$ from Lam9\% to Lam9\%thinner2, leading to Reynolds numbers $\operatorname{Re}_{\theta}=u_{j} \delta_{\theta} / v$ ranging between 781 and 290.

(a)

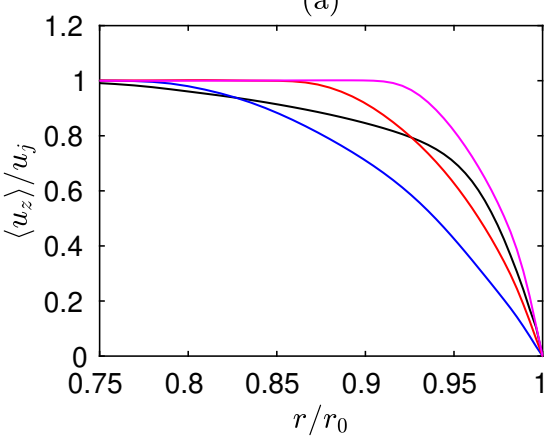

(b)

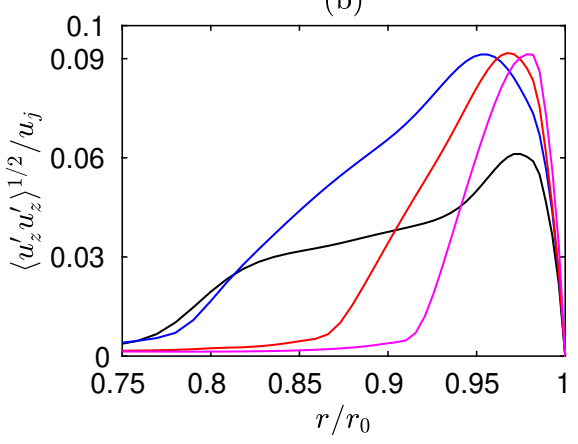

Fig. 3 Profiles of the (a) mean and (b) rms values of axial velocity at the nozzle exit: Lam9\%,_ Lam9\%thinner1, —— Lam9\%thinner2.

Turb6\%,

In figure 3 (b), the profiles of turbulence intensity are similar to those measured for jets with upstream boundary layers in highly-disturbed laminar [2] or turbulent [14, 23] states. The peak intensities caused by the boundary-layer forcing are found to be $u_{e}^{\prime} / u_{j}=6.12 \%$ for Turb6\% and between $u_{e}^{\prime} / u_{j}=9.13 \%$ and $9.17 \%$ for the other jets. Therefore, Lam9\%, Lam9\%thinner1 and Lam9\%thinner2 are initially nominally laminar jets which, at the nozzle 
Table 1 Nozzle-exit flow conditions and peak Strouhal numbers of instability waves at $z=0.1 r_{0}$.

\begin{tabular}{cccccccc}
\hline \hline jet & $\mathrm{H}$ & $\delta_{\theta} / r_{0}$ & $\delta_{\omega} / r_{0}$ & $\mathrm{Re}_{\theta}$ & $u_{e}^{\prime} / u_{j}$ & $\mathrm{St}_{D}$ & $\mathrm{St}_{\theta}$ \\
\hline Turb6\% & 1.71 & 0.0274 & 0.043 & 685 & 6.12 & 2.31 & 0.0321 \\
Lam9\% & 2.19 & 0.0313 & 0.099 & 781 & 9.13 & 1.14 & 0.0184 \\
Lam9\%thinner1 & 2.24 & 0.0186 & 0.058 & 465 & 9.17 & 2.03 & 0.0197 \\
Lam9\%thinner2 & 2.13 & 0.0116 & 0.034 & 290 & 9.13 & 3.02 & 0.0189 \\
\hline \hline
\end{tabular}

exit, have stronger velocity fluctuations and boundary layers of similar and smaller momentum thickness compared to Turb6\%. This is in line with the conditions measured downstream of the ASME/conical nozzles [12]. For the sake of completeness, the vorticity thicknesses evaluated from the maximum values of velocity gradient at the nozzle exit are provided in table 1. They are equal to $\delta_{\omega}=0.043 r_{0}$ for Turb6\%, and to $\delta_{\omega}=0.099 r_{0}$ for Lam9\%, $0.058 r_{0}$ for Lam9\%thinner1 and $0.034 r_{0}$ for Lam9\%thinner2. Thus, the exit boundary layer of Turb6\% has a momentum thickness close to that of Lam9\%, but a vorticity thickness between those of Lam9\%thinner1 and Lam9\%thinner2.

The effects of the nozzle-exit turbulence levels on the jet with a non-laminar boundary-layer profile are not examined in this paper. However, the cases of two jets with untripped boundary layers [24], denoted as Turb0\% and Lam0\%, are reported in an appendix. At the exit of the nozzle-pipe, these jets are characterized by mean velocity profiles very similar to those for Turb6\% and Lam6\%, respectively, and peak turbulence intensities $u_{e}^{\prime} / u_{j} \ll 1 \%$.

\section{B. Numerical methods and details}

The numerical methods and parameters used in the jet large-eddy simulations and in the extrapolations of the LES near-field fluctuations to the acoustic far field are described in depth in the paper [17] where Turb6\% was previously considered.

The LES are carried out using an in-house solver of the three-dimensional filtered compressible Navier-Stokes equations in cylindrical coordinates $(r, \theta, z)$, based on finite-difference and Runge-Kutta explicit schemes with low dissipation and low dispersion [25-27]. A six-order explicit filtering is applied every time step in order to damp gridto-grid oscillations, but also as an LES subgrid-scale model relaxing turbulent energy from scales at wave numbers close to the grid cut-off wave number while leaving larger scales mostly unaffected [28, 29]. At the grid boundaries, radiation conditions [30] are implemented with the addition of a sponge zone combining grid stretching and Laplacian filtering at the outflow. At the inflow and radial boundaries, density and pressure are also brought back close to $p_{a}$ and $\rho_{a}$, in order to keep the mean values of density and pressure around their ambient values without generating significant acoustic reflections. No co-flow is imposed.

The jet simulations are performed on a mesh grid containing $N_{r} \times N_{\theta} \times N_{z}=504 \times 1024 \times 2048=1.1 \times 10^{9}$ points, extending radially out to $L_{r}=15 r_{0}$ and axially, excluding the 100-point outflow sponge zone, down to $L_{z}=40 r_{0}$. The mesh spacing is minimum and equal to $0.0036 r_{0}$ at $r=r_{0}$ along the nozzle-lip line, and is maximum and is equal to $0.075 r_{0}$ over $6.25 r_{0} \leq r \leq L_{r}$, yielding a Strouhal number of $\mathrm{St}_{D}=f D / u_{j}=5.9$ for an acoustic wave discretized by five points per wavelength, where $f$ is the frequency. The properties and quality of the grid have been exhaustively discussed in the reference [31] reporting a grid-sensitivity study of the flow and sound fields for a Mach number 0.9 jet at $\operatorname{Re}_{D}=10^{5}$ with a Blasius boundary-layer profile of thickness $\delta_{B L}=0.15 r_{0}$ and a peak turbulence intensity of $u_{e}^{\prime} / u_{j}=9 \%$ at the nozzle exit. For Turb6\% with a non laminar boundary-layer profile, the near-wall mesh spacings in the radial, azimuthal and axial directions are equal to 2.7, 4.6 and 5.4, in wall units, respectively. The use of a radial mesh spacing twice as small at the wall inside the pipe was shown not to affect the mean and turbulent profiles at the pipe exit in reference [17]. For the three jets with laminar boundary-layer profiles, the velocity spectra just downstream of the nozzle were found not to appreciably depend on the grid resolution in reference [22].

For the four jets, the simulation time after the transient period is equal to $500 r_{0} / u_{j}$. During that time period, the signals of density, velocities and pressure obtained on the jet axis at $r=0$, on the cylindrical surfaces located at $r=r_{0}$ and $r=L_{r}=15 r_{0}$ and in the cross sections at $z=-1.5 r_{0}, z=0$ and $z=L_{z}=40 r_{0}$, are recorded at a sampling frequency allowing spectra to be computed up to $\mathrm{St}_{D}=12.8$. The signals obtained in the four azimuthal planes at $\theta=0, \pi / 4, \pi / 2$ and $3 \pi / 4$ are also stored, at a halved frequency in order to reduce storage requirements. The flow statistics presented in the next section are calculated from these recordings. Time spectra are evaluated from overlapping samples of duration $90 r_{0} / u_{j}$. 
The jet near-field fluctuations are propagated to the far field using an in-house OpenMP-based solver of the isentropic linearized Euler equations (ILEE) in cylindrical coordinates, based on the same numerical methods as the LES. The extrapolations are carried out from the LES velocity and pressure fluctuations at $r=15 r_{0}$ and at $z=-1.5 r_{0}$ and $40 r_{0}$, using grids containing up to $1.6 \times 10^{9}$ points with $N_{\theta}=256$ and a uniform mesh spacing of $0.075 r_{0}$ in the axial and radial directions. They allow us to obtain the pressure waves radiated at a distance of $150 r_{0}$ from the nozzle exit, where far-field acoustic conditions are expected to apply according to measurements [32], between the angles of $\phi=15^{\circ}$ and $\phi=165^{\circ}$ relative to the jet direction.

Finally, as was done in previous investigations [14, 16, 17], an inviscid spatial stability analysis is conducted from the jet mean flow fields in the present work. In practice, for a given axial distance $z$ between $z=0.02 r_{0}$ and $5 r_{0}$ and for a given Strouhal number $\mathrm{St}_{D}$, the compressible Rayleigh equation [33] is solved through a shooting technique [34], based on the Euler method for the integration step and on the secant method for the search of the complex wavenumber $k_{z}$. The integration is performed directly from the LES mean flow profiles, interpolated on a grid extending from $r=0$ to $3 r_{0}$ every $0.0005 r_{0}$ and then smoothed using a high-order centered filter in order to remove spurious high-frequency oscillations.

\section{Results}

\section{A. Vorticity and pressure snapshots}

Instantaneous fields of the square of vorticity norm obtained down to $z=3.5 r_{0}$ for the four jets are represented in figure 4. Very near the nozzle lip, structures elongated in the downstream direction, characteristic of wall bounded flows, are found. Their length scales in the radial direction are of the order of boundary-layer vorticity thickness, and are therefore smaller for Turb6\% and Lam9\%thinner2 than for the two other jets. Farther from the nozzle, the shear-layers roll up between $z=0.5 r_{0}$ and $z=r_{0}$, and typical mixing-layer structures are then generated. For Turb6\%, these structures are rapidly much larger than the boundary-layer structures, compare for instance the turbulent length scales at $z=r_{0}$ and $z=2 r_{0}$ in figure 4(a). For the three other jets, the growth of the vortical structures downstream of the nozzle is more gradual. Similar results are observed for Turb0\% and Lam0\% in figure 15 of the appendix. In figures 4(c,d), large-scale structures can also be seen for Lam9\%thinner1 and Lam9\%thinner2 despite the high levels of velocity fluctuations at the nozzle-exit.
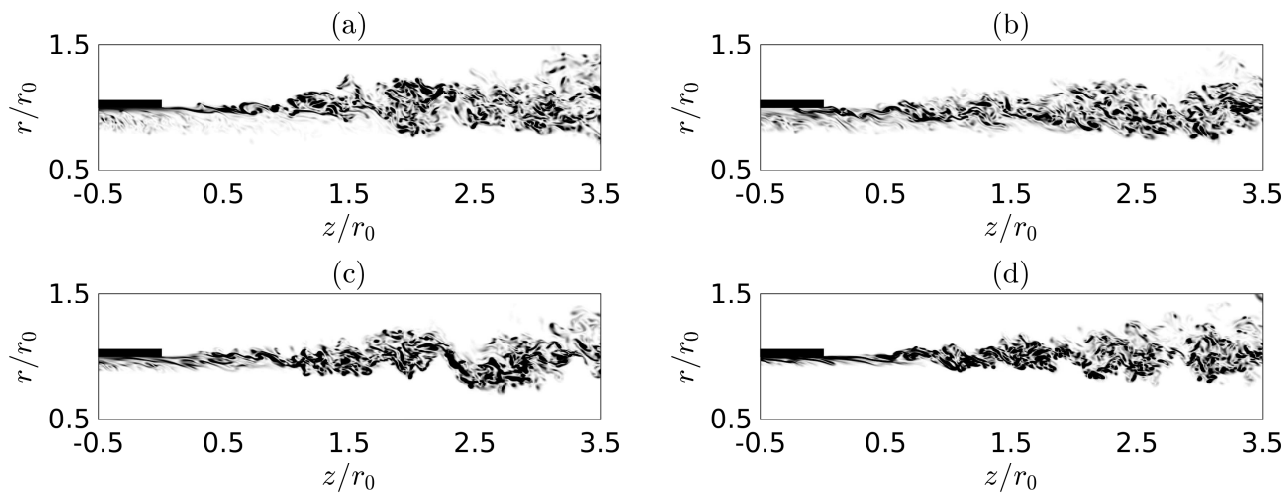

Fig. 4 Square of vorticity norm for (a) Turb6\%, (b) Lam9\%, (c) Lam9\%thinner1 and (d) Lam9\%thinner2. The gray scale varies from 0 up to $350 \times\left(u_{j} / r_{0}\right)^{2}$.

Snapshots of the flow fields are provided in figure 5 for Turb6\% and Lam9\%thinner1 down to $z=10 r_{0}$. For Lam9\%thinner1, large-scale turbulent structures of high vorticity level, of size comparable to the transverse length scale of the shear layer and spatially isolated from each other [35, 36], clearly appear in figure 5 (b). These structures are very similar to the coherent structures of the flow visualizations of Brown \& Roshko [37]. They can be detected at the axial positions of $z=2 r_{0}, 4.5 r_{0}, 6.5 r_{0}$ and $9 r_{0}$, for example, suggesting that they persist over a long distance from the nozzle. For Turb6\%, on the contrary, the presence of coherent structures is not obvious in figure 5 a). These results are consistent with previous studies on initially nominally laminar jets [15, 17].

The pressure fields of the four jets are represented in figure 6 down to $z=8 r_{0}$, in order to focus on the mixing layers and their radiated noise. Spurious high-frequency acoustic waves due to the inlet forcing are visible in the 
(a)

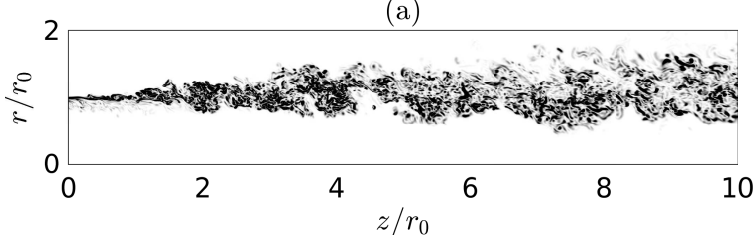

(b)

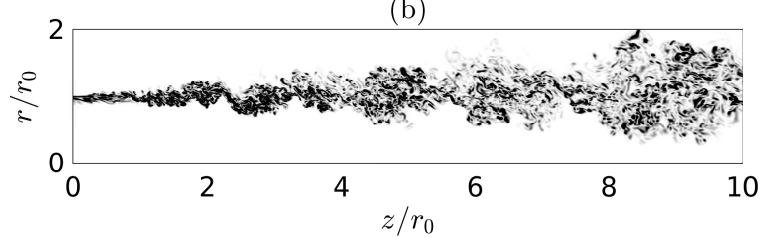

Fig. 5 Square of vorticity norm for (a) Turb6\% and (b) Lam9\% thinner1. The gray scale varies from 0 up to $175 \times\left(u_{j} / r_{0}\right)^{2}$.

vicinity of the nozzle exit in figures 6(a,b) for Turb6\% and Lam9\%. Such waves do not emerge for Lam9\%thinner1 and Lam9\%thinner 2 because they are rapidly damped due to their shorter wavelength, itself proportional to the boundary-layer thickness [17, 19]. More interestingly, hydrodynamic fluctuations, associated with the flow large-scale structures, dominate in and very near the jets, as expected [38]. They may seem more organized for the three jets with laminar boundary-layer profiles than for Turb6\%, especially for Lam9\%thinner1 in figure 6(c). This further supports the presence of more coherent turbulent structures in the first three jets. Outside the jet flows, the sound waves are of similar amplitude for Turb6\% and Lam9\%, but stronger for Lam9\% thinner1 and Lam9\%thinner2 with thinner boundary layers. This is particularly the case for the high-frequency waves generated between $z=2 r_{0}$ and $z=4 r_{0}$ during the earlier stage of development of the mixing layers, see in figure 6(d) for instance.
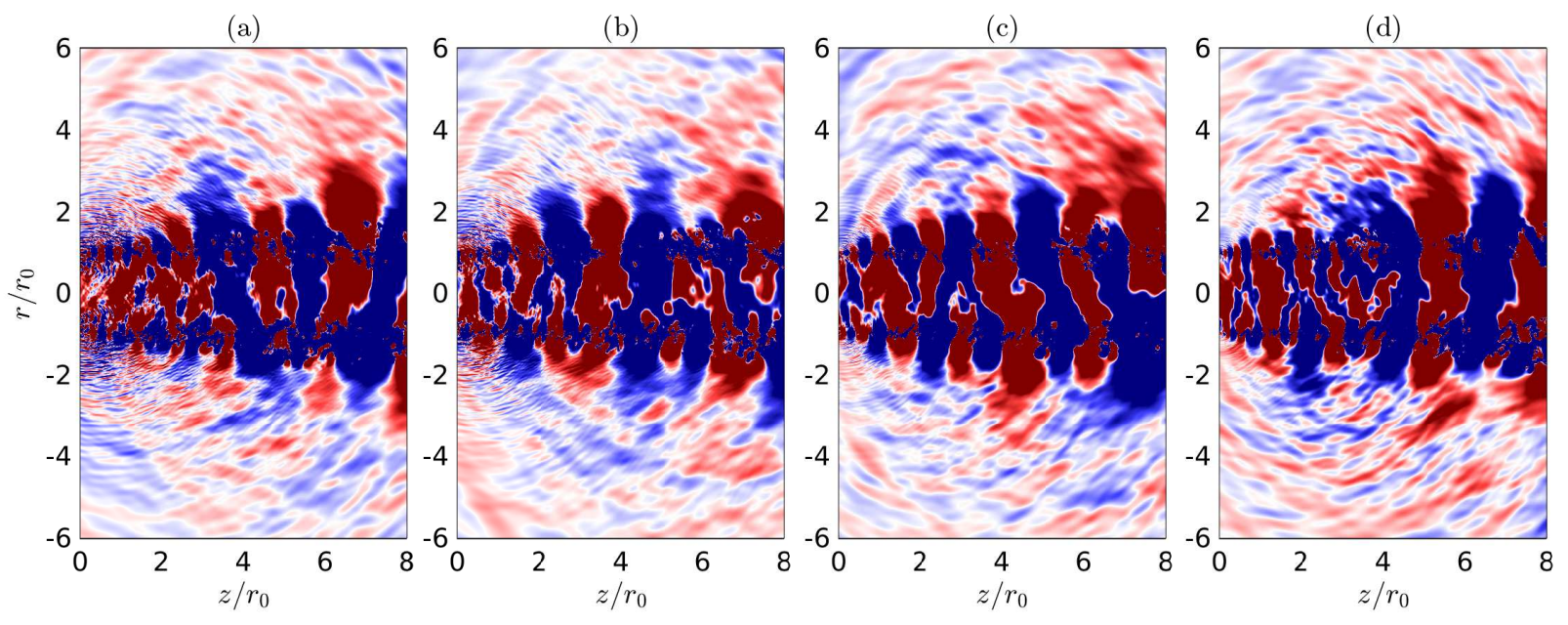

Fig. 6 Pressure fluctuations for (a) Turb6\%, (b) Lam9\%, (c) Lam9\%thinner1 and (d) Lam9\%thinner2. The color scale varies between $\pm 1.8 \times 10^{-3} p_{a}$, from blue to red.

\section{B. Jet instability waves and flow fields}

The instability growth rates $-\operatorname{Im}\left(k_{z}\right) r_{0}$ computed for the axisymmetric mode from the LES mean flow profiles at $z=0.1 r_{0}$ using the inviscid linear stability analysis mentioned in section II.B are represented in figure7 7 a) as a function of the Strouhal number $\mathrm{St}_{D}$. The axisymmetric mode is considered because it is the dominant one, but very similar results are found for the first helical modes [33]. For the initially nominally laminar jets, the growth rates strengthen and the range of unstable frequencies broadens as the thickness of the boundary-layer profile decreases, yielding peak Strouhal numbers of $\mathrm{St}_{D}=1.14$ for Lam9\%, 2.03 for Lam9\%thinner1 and 3.02 for Lam9\%thinner2, as documented in table 1 For Turb6\%, the amplification curve stands between those for Lam9\%thinner1 and Lam9\%thinner2, and is at its maximum at $\mathrm{St}_{D}=2.31$.

The influence of the boundary-layer profile on the jet initial unstable frequencies is better illustrated by plotting the instability growth rates at $z=0.1 r_{0}$ as a function of the Strouhal number $\mathrm{St}_{\theta}=f \delta_{\theta} / u_{j}$ in figure 7(b). The amplification curve determined for a hyperbolic-tangent profile with $\delta_{\theta}=0.029 r_{0}$, corresponding to the momentum thickness imposed at the pipe-nozzle inlet for Lam9\% and Turb6\%, is also depicted. This analytical profile is often 
used in linear stability analyses for mixing layers and jets [33, 39], because it gives a good approximation of the mean velocity distribution in such flows [40,41]. This velocity distribution itself does not depend on the laminar or turbulent state of the initial boundary layers [42]. For the three jets with laminar boundary-layer profiles, the maximum growth rates are reached at Strouhal numbers around $\mathrm{St}_{\theta}=0.019$, see in table 1. This value is comparable with the peak Strouhal number of $\mathrm{St}_{\theta}=0.017$ obtained for the hyperbolic-tangent profile. In contrast, for Turb6\%, the growth rate is maximal at $\mathrm{St}_{\theta}=0.032$. Thus, the most unstable frequencies just downstream of the nozzle are close to those expected farther downstream in the mixing layers for the three nominally laminar jets, but they are significantly higher for Turb6\%.

(a)

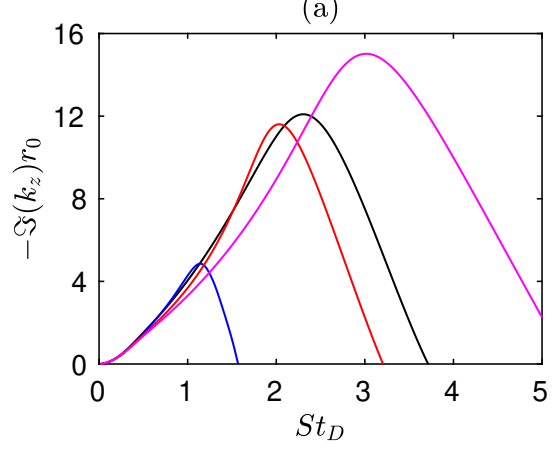

(b)

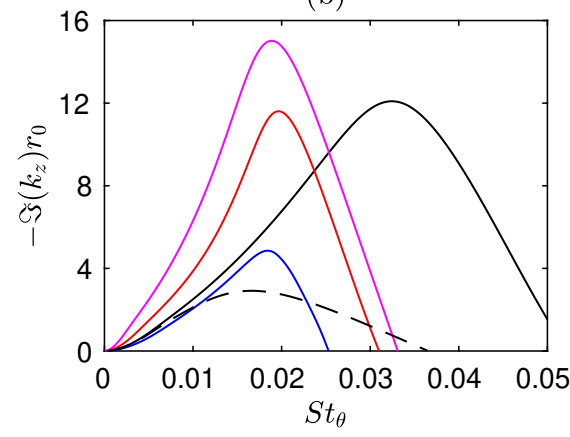

Fig. 7 Instability growth rates at $z=0.1 r_{0}$ as a function of (a) $\mathrm{St}_{D}$ and (b) $\mathrm{St}_{\theta}$ : - $-\mathrm{Turb6 \%}$, Lam9\%, — Lam9\% thinner1, — Lam9\%thinner2; - - - 2-D hyperbolic-tangent velocity profile.

The spectra of radial velocity fluctuations calculated at $r=r_{0}$ and $z=0.2 r_{0}$ are represented in figure 8 as a function of $\mathrm{St}_{D}$. The peak Strouhal numbers obtained from the mean flow profiles at $z=0.1 r_{0}$ using the inviscid linear stability analysis are also indicated. For all jets, despite the highly-disturbed boundary-layer flow conditions at the nozzle exit, the spectra are dominated by components at frequencies in good agreement with those estimated by the stability analysis. Therefore, the flow development near the nozzle is intrinsically linked to the instability waves growing in the exit boundary-layer profiles. It can be noted that the peak intensities in figure 8 do not vary as the maximum instability growth rates in figure 7 (a). This can be attributed to viscous effects, which are not taken into account in the stability analysis, but can be expected to be non negligible in Lam9\%thinner1 and Lam9\%thinner2 at Reynolds numbers $\operatorname{Re}_{\theta}<500$ [34, 43], and in Turb6\% due the high velocity gradient in the boundary-layer profile in that case.

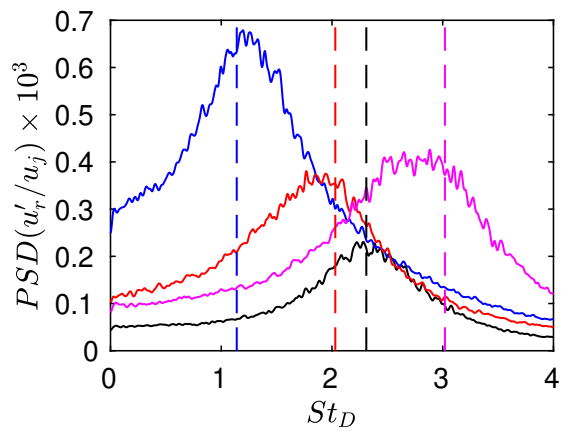

Fig. 8 Spectra of radial velocity at $r=r_{0}$ and $z=0.2 r_{0}:-$ Turb6\%, - - Lam9\%, Lam9\% thinner1, — Lam9\%thinner2; dashed lines: peak frequencies of instability waves at $z=0.1 r_{0}$.

In order to highlight the spatial evolution of the instability waves early on in the shear layers, the growth rates obtained between $z=0.05 r_{0}$ and $3 r_{0}$ from the linear stability analysis are displayed in figure 9 for Turb6\%, Lam9\%thinner1 and Lam9\%thinner2. Contour lines are drawn for the levels of $-\mathfrak{J}\left(k_{z}\right) r_{0}=0.1,0.6,2,6$ and 12 . The Strouhal numbers of the peak growth rates at $z=0.1 r_{0}$ and $0.2 r_{0}$ are also shown. For all jets, unsurprisingly, the most unstable frequency decreases in the axial direction as the mixing layer develops and becomes thicker. Looking more specifically at the dominant instability waves at $z=0.1 r_{0}$ and $0.2 r_{0}$, they appear to be amplified down to $z \simeq 0.7 r_{0}$ in 
figure 9a) for Turb6\%, $z \simeq 1.8 r_{0}$ in figure 9b) for Lam9\%thinner1 and $z \simeq 1.3 r_{0}$ in figure 9 (c) for Lam9\%thinner2. The initial instability waves thus continue to grow over a longer distance from the nozzle in Lam9\%thinner1 and Lam9\%thinner2 than in Turb6\%, despite thinner boundary layers. This is true, in particular, for Lam9\%thinner1 for which the amplification curve at $z=0.1 r_{0}$ is very similar to that for Turb6\% in figure 7 a). Therefore, the difference in persistence of the initial instability waves cannot be related to different instability growth rates, due for instance to different maximum gradients in the velocity profiles. On the contrary, as suggested previously [17], it results from the fact that for a laminar boundary-layer profile, the instability frequencies are comparable to those expected downstream in the mixing layer, whereas for a non-laminar profile they do not match and are higher than the shear-layer instability frequencies as can be seen in figure 7 b).

(a)

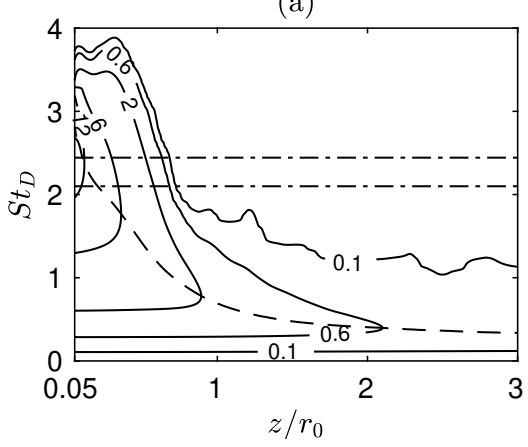

(b)

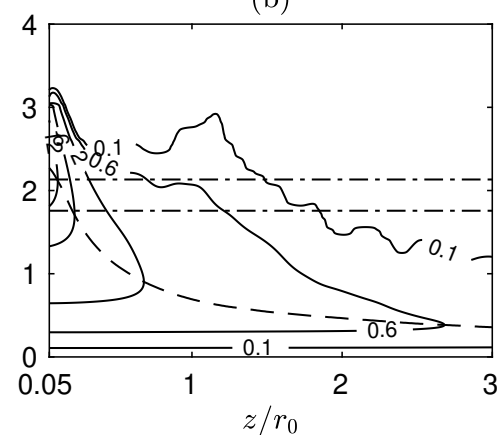

(c)

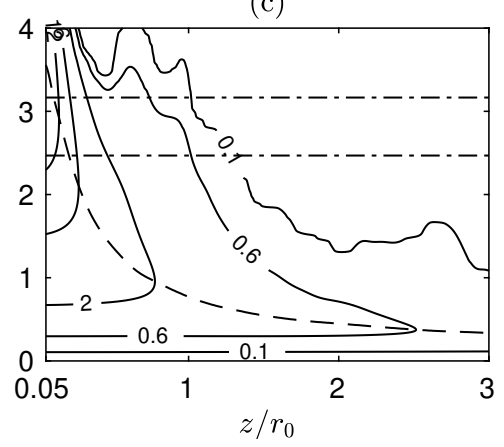

Fig. 9 Instability growth rates $-\mathfrak{J}\left(k_{z}\right) r_{0}$ as a function of $z$ and $\mathrm{St}_{D}$ : (a) Turb6\%, (b) Lam9\%thinner1, (c) Lam9\%thinner2; - - most unstable frequencies; $-\cdots$ peak frequencies at $z=0.1 r_{0}$ and $z=0.2 r_{0}$.

The spectra of radial velocity fluctuations obtained at $r=r_{0}$ and $z=0.8 r_{0}, 1.6 r_{0}, 3.2 r_{0}, 4.8 r_{0}, 6.4 r_{0}$ and $8 r_{0}$ are represented in figure 10 as a function of $\mathrm{St}_{D}$, along with the peak frequencies of instability growth rates at $z=0.1 r_{0}$. At $z=0.8 r_{0}$ in figure 10 a), the spectra are dominated by humps associated with the initial instability waves, centered around Strouhal numbers slightly lower than those predicted at $z=0.1 r_{0}$ due to the shear-layer thickening. As the axial distance increases, the spectra become progressively broadband as turbulence develops in the mixing layers, in all cases. However, instability-wave components remain noticeable over a larger or shorter distance.

For Turb6\%, the spike emerging around $\mathrm{St}_{D} \simeq 2$ at $z=0.8 r_{0}$ in figure 10 (a) no longer appears at $z=1.6 r_{0}$ in figure 10 b), and the velocity spectra are all broadband for $z \geq 3.2 r_{0}$ in figures 10(c-f). For the initially nominally laminar jets, instability-wave components are visible down to $z=3.2 r_{0}$ in figure 10 (c) for Lam9\%thinner2, $z=4.8 r_{0}$ in figure 10 (d) for Lam9\%thinner1, and $z=6.4 r_{0}$ in figure 10(e) for Lam9\%, that is much farther downstream than for Turb6\%. These results are consistent with the above linear stability analysis. They show that despite stronger initial flow disturbances, the jets with laminar boundary-layer profiles contain more organized (or coherent) turbulent structures than the jet with a non-laminar profile, in agreement with the vorticity fields of figure 5 and the observations made in previous studies [15, 17]. Higher turbulence levels are also found at low Strouhal numbers, typically below $\mathrm{St}_{D}=0.6$, as for example in the spectra at $z=8 r_{0}$ of figure 10 (f).

The spreading rates $\mathrm{d} \delta_{\theta} / \mathrm{d} z$ of the jet shear layers are presented in figure 11 between $z=0$ and $10 r_{0}$. Very near the nozzle exit, they rapidly grow and reach values at $z \simeq 1.5 r_{0}$ depending on the maximum velocity gradient in the exit boundary-layer profiles. For the initially nominally laminar jets, the spreading rates around this position are higher as the boundary layer is thinner. For Turb6\%, they are similar to those for Lam9\%thinner2, although spreading rates between those for Lam9\%thinner 1 and Lam9\% thinner 2 could have been expected according to the vorticity thicknesses reported in table1. This may be due to the lower initial turbulence level in Turb6\%, which is likely to strengthen the early stage of mixing-layer development compared to the other jets [8]. Farther downstream, the spreading rates continue to increase for Lam9\%, remain high for Lam9\%thinner1 and Lam9\%thinner2, and significantly decrease for Turb6\%. As a consequence, for $z \geq 4 r_{0}$, they are stronger for the initially nominally laminar jets than for Turb6\%. Considering the major role played by coherent structures in the entrainment of the surrounding fluid into the jet flow [35, 37, 44], hence in the mixing-layer spreading, this can be attributed to the presence of more structures of this kind in Lam9\%, Lam9\%thinner1 and Lam9\% thinner2 than in Turb6\%.

Finally, the variations of the rms values of axial and radial velocity fluctuations obtained along the nozzle-lip line are shown in figure 12 down to $z=15 r_{0}$, corresponding approximately to the end of the jet potential core. They bear striking similarities to the variations of the shear-layer spreading rates. Just downstream of the nozzle, the increase 
(a)

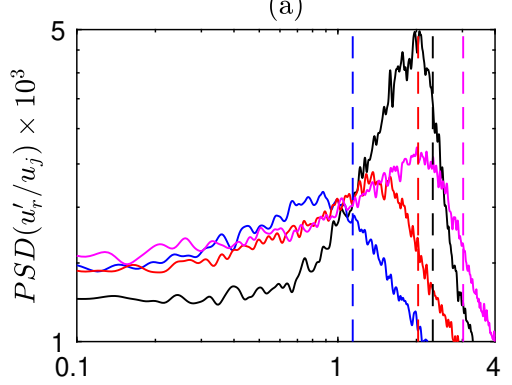

(d)

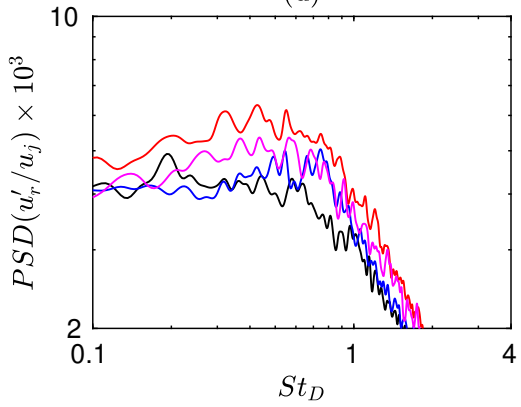

(b)

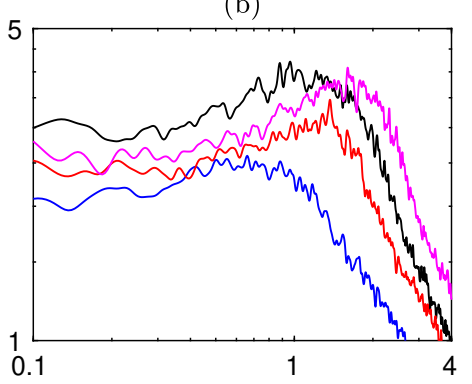

(e)

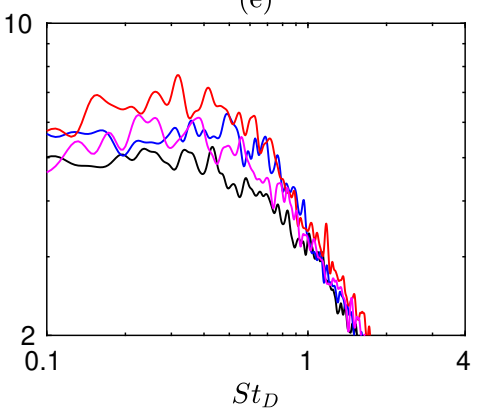

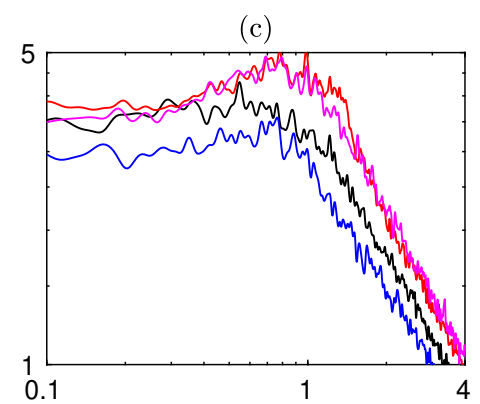

(f)

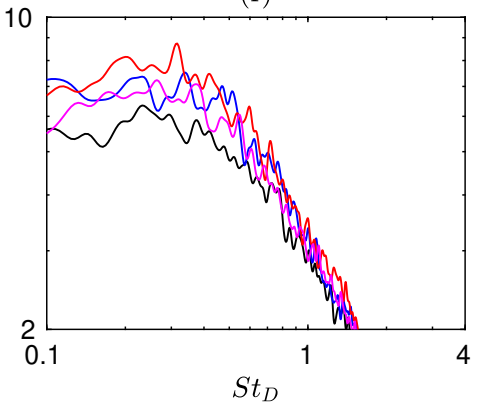

Fig. 10 Spectra of radial velocity at $r=r_{0}$ at (a) $z=0.8 r_{0},(\mathrm{~b}) z=1.6 r_{0},(\mathrm{c}) z=3.2 r_{0}$, (d) $z=4.8 r_{0}$, (e) $z=6.4 r_{0}$ and (f) $z=8 r_{0}$ : — Turb6\%, — Lam9\%, — Lam9\%thinner1, — Lam9\%thinner2; dashed lines: peak frequencies of instability waves at $z=0.1 r_{0}$.

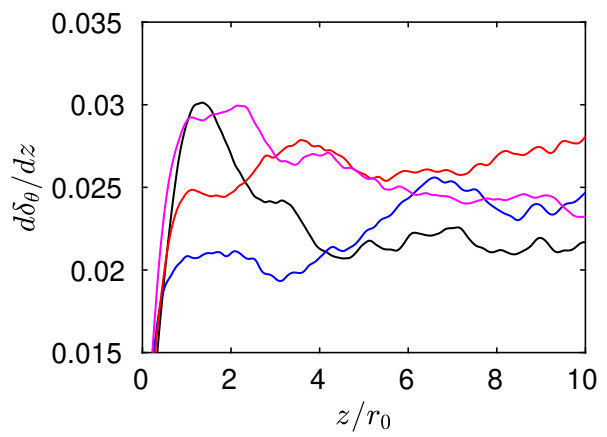

Fig. 11 Variations of the shear-layer spreading rate:

Turb6\% Lam9\%, Lam9\%thinner1, Lam9\%thinner2. 
of the turbulence intensities is faster and leads to higher levels as the vorticity thickness of the exit velocity profile is smaller, refer also to table 1 . For $z \geq 5 r_{0}$, however, the rms values of velocity fluctuations are larger for the initially nominally laminar jets than for Turb6\%. This result is not surprising given the spectra of figure 10 containing stronger instability-wave and large-scale components for the former jets.

(a)

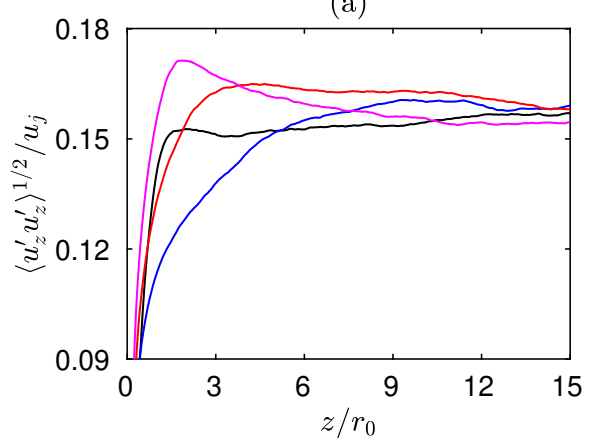

(b)

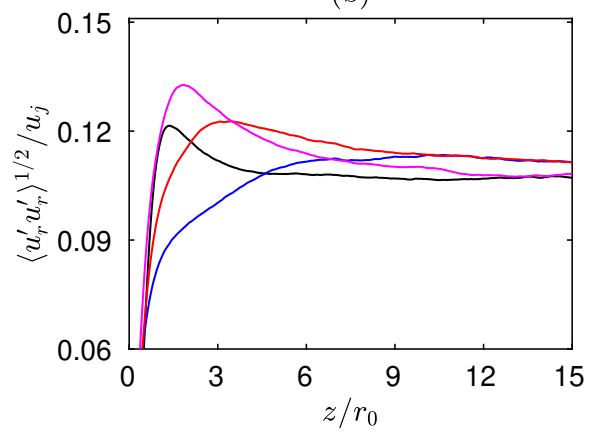

Fig. 12 Variations of the rms values of (a) axial and (b) radial velocities at $r=r_{0}:-$ Turb6\%, Lam9\%, — Lam9\%thinner1, — Lam9\%thinner2.

For the three jets with laminar boundary-layer profiles, the higher peak turbulence intensities reached for a thinner boundary layer can be related to the decrease of the Reynolds number $\operatorname{Re}_{\theta}$ [21, 22]. It is also interesting to compare the rms axial velocities obtained for Lam6\% and Lam9\%, which are represented in blue in figures 2 a) and 12 (a). In line with previous studies [8], increasing the value of $u_{e}^{\prime} / u_{j}$ from $6 \%$ to $9 \%$, with all other parameters being held constant, significantly reduces the rms levels of velocity in the mixing layers. Despite this, the rms levels for Lam9\% still exceed those for Turb6\%, suggesting that the effects of the boundary-layer profile are greater than the effects of the initial turbulence in this case.

\section{Acoustic far fields}

The overall sound pressure levels obtained in the acoustic far field at 150 radii from the nozzle exit are represented in figure 13 between the angles $\phi=15^{\circ}$ and $\phi=150^{\circ}$ relative to the jet direction. For all angles, they are higher for the jets with laminar boundary-layer profiles than for Turb6\%. The level increase, however, varies depending on the jet exit conditions and the radiation angle. In the downstream direction, for the angle $\phi=30^{\circ}$ of peak noise emission, it is approximately of $2 \mathrm{~dB}$ for Lam9\% and Lam9\%thinner2 and of $3 \mathrm{~dB}$ for Lam9\%thinner1. In the sideline and upstream directions, for $\phi \geq 60^{\circ}$, it does not exceed $1 \mathrm{~dB}$ for Lam9\% and is close to $2 \mathrm{~dB}$ for Lam9\%thinner1 and Lam9\%thinner2. Following experimental studies [45], the excess noise of the initially nominally laminar jets with respect to the non-laminar case can be linked to the higher rms values of velocity fluctuations in the jet mixing layers, revealed in figure 12 It is stronger for Lam9\%thinner1 and Lam9\%thinner2 than for Lam9\%, that is, when the boundary layer is thinner compared to the non-laminar case, in particular for large radiation angles. However, the sound levels for Lam9\%thinner2 are slightly lower than those for Lam9\%thinner1. This may be due to the fact that in the former case, the effects of the laminar exit mean velocity profile on the mixing-layer turbulence, and hence on the sound sources, happen over a shorter distance in the axial direction, see again in figure 12

The pressure spectra evaluated in far field for the angles of $\phi=30^{\circ}$ and $90^{\circ}$ are represented in figure 14 as a function of $\mathrm{St}_{D}$. Due to the difference in shape of the spectra at these two angles, characteristic of subsonic jet noise [1, 46], they are displayed using a range of $30 \mathrm{~dB}$ with a $6 \mathrm{~dB}$ step for $\phi=30^{\circ}$, but only of $15 \mathrm{~dB}$ with a $3 \mathrm{~dB}$ step for $\phi=90^{\circ}$. With respect to Turb6\% with a non-laminar boundary-layer profile, the sound levels for Lam $9 \%$ with nearly the same boundary-layer momentum thickness are stronger for $\mathrm{St}_{D} \leq 0.6$, but comparable for higher Strouhal numbers. In contrast, more noise is noticed up to $\mathrm{St}_{D}=4$ in figure 2 b) for Lam6\%. Therefore, increasing the nozzle-exit turbulence level $u_{e}^{\prime} / u_{j}$ from $6 \%$ to $9 \%$ is sufficient to make the additional noise due to the laminar boundary-layer profile disappear at high frequencies but not at low frequencies in this case. The dominant acoustic components around $\mathrm{St}_{D}=0.25$ at $\phi=90^{\circ}$ are nevertheless reduced significantly, as can be seen from the blue lines in figures 2 (b) and 14 (b). For higher nozzle-exit turbulence intensities, such as $u_{e}^{\prime} / u_{j}=12 \%$ or $15 \%$ in Lam $12 \%$ or Lam $15 \%$ jets for instance, they could be expected to vanish [8], leading to noise levels closer to or possibly lower than those for Turb6\%. In the same way, at the lower end of the range of the initial turbulence intensities, the acoustic radiation is found to be stronger 


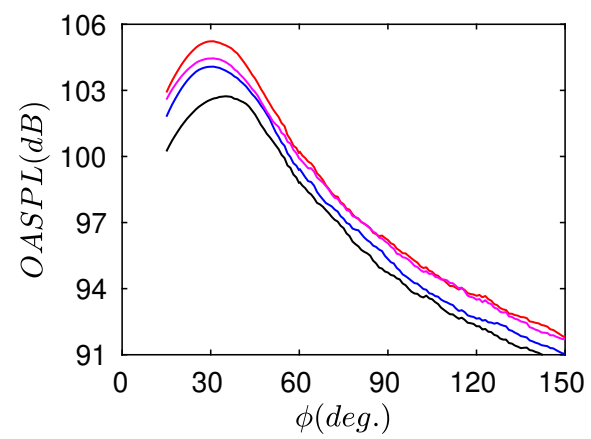

Fig. 13 Far-field overall sound pressure levels as a function of the radiation angle: Lam9\%, Lam9\%thinner1, Lam9\%thinner2.

Turb6\%,

for Lam0\% than for Turb0\% but weaker for Lam6\% in the appendix.

For Lam9\%thinner1 and Lam9\%thinner2, the sound levels are higher than those for Turb6\% at low frequencies but also, contrary to Lam9\%, at high frequencies. The emergence of extra acoustic components at such frequencies for these two jets most likely results from their thinner nozzle-exit boundary layers. Obviously, it can be related to the larger velocity fluctuations observed in figure 12 early on in the mixing layers, where high-frequency noise is generated [47-49]. It can also be correlated with the strong mid- and high-frequency components appearing in figure 10 (c) in the velocity spectra computed at $r=r_{0}$ and $z=3.2 r_{0}$, at a position around which intense sound waves are emitted according to figures 6(c,d). Comparing Lam9\%thinner2 to Lam9\%thinner1 in figure 14(b), more noise is obtained for Lam9\%thinner1 at $\mathrm{St}_{D} \leq 0.6$, and for Lam9\%thinner2 at $\mathrm{St}_{D} \geq 1$. Therefore, decreasing the boundary-layer thickness for a laminar profile not only strengthens the excess noise at high frequencies, but can also make it weaker at low frequencies. On this basis, it can be assumed that for a hypothetical Lam9\%thinner3 case with a boundary-layer thickness $\delta_{\theta} \simeq 0.05 r_{0}$ close to that in the jets from the ASME nozzle [17], the excess noise would be stronger at high frequencies than at low frequencies.

It is finally worth reexamining the experimental results available in the literature for subsonic jets with initially highly-disturbed laminar and turbulent boundary-layer profiles [11-14] in the light of the findings of the present work. These jets have Reynolds numbers typically of one order of magnitude higher and boundary layers 3-4 times thinner with respect to the simulated jets, refer to the table 1 in Bogey \& Sabatini [17]. Despite this, a great similarity can be found between their far-field sound spectra and those in figure 14. This is particularly true for the spectra of figure 1 , acquired for jets with laminar and turbulent boundary layers, which are more disturbed and thinner in the former case than in the latter according to Zaman [12], such as for Lam9\%thinner1 and Lam9\%thinner2 compared to Turb6\%. This suggests that the excess noise measured for initially nominally laminar jets, using the ASME nozzle for instance, is of same nature and has the same physical explanation as that described in this study. It appears however at higher frequencies because of the much thinner boundary layers in the experiments than in the simulations, as discussed above.

(a)

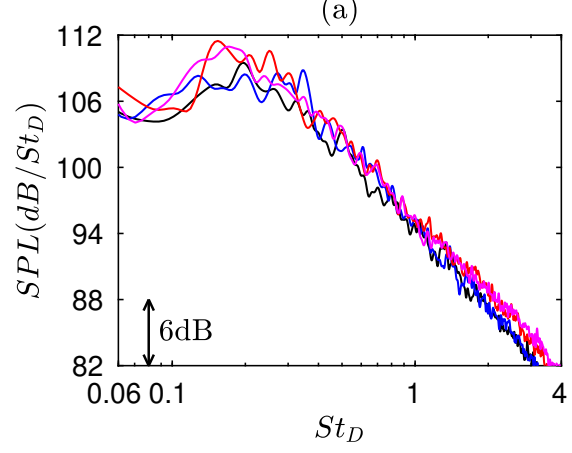

(b)

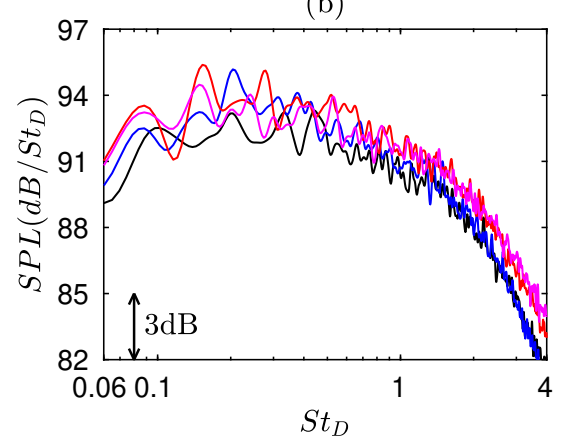

Fig. 14 Far-field sound pressure levels for (a) $\phi=30^{\circ}$ and (b) $\phi=90^{\circ}$ : — Turb6\%, - - Lam9\%, Lam9\%thinner1, Lam9\%thinner2. 


\section{Conclusion}

In this paper, the generation of noise by initially nominally laminar jets, namely jets with highly-disturbed Blasius laminar boundary-layer profiles, is investigated using large-eddy simulations. For that purpose, a set of four subsonic jets with well-controlled nozzle-exit conditions is considered. The jets are at a moderate Reynolds number in order to ensure a high numerical accuracy, and have laminar or non-laminar boundary-layer profiles. Larger velocity fluctuations are imposed at the nozzle exit in the laminar case, as happens in some experiments [9, 12]. The three jets with laminar boundary-layer profiles generate higher sound levels than the other one. This result may seem counter-intuitive because more noise could have been expected in the jet with a non-laminar boundary-layer profile which is initially less disturbed. However, it is consistent with experimental trends, especially with those obtained using ASME/conical nozzles [11-13]. The level increase observed at high frequencies moreover appears to be due to the thinner boundary layers of the initially nominally laminar jets.

The present work confirms the significant influence of the shape of the boundary-layer profile on the flow and sound fields of subsonic jets. It also highlights the key role played in this regard by the difference in frequency of the instability waves growing near the nozzle exit depending on the boundary-layer profile. As reported in a recent work [17], for a laminar profile, the most unstable frequencies are similar to those obtained farther downstream in the mixing-layer profiles, whereas they are much higher for a non-laminar profile. This leads to instability waves amplified over a longer distance, more organized coherent turbulent structures in the mixing layers, and stronger turbulence intensities and radiated sound waves. These effects are strong and can exceed those caused by the variations of other jet initial parameters, such as the nozzle-exit velocity fluctuation levels in some cases.

Given the findings of the present study, it can be interesting to remind the reader of some issues encountered experimentally when the initial shear layer of jets is artificially excited at a single frequency. This has been found to result to a suppression [50, 51] or an amplification [52] of the broadband components of flow turbulence and/or farfield noise. These two responses to excitation have been attributed to the laminar or turbulent states of the nozzle-exit boundary layers [53, 54]. They may be linked to the different shapes of the boundary-layer profiles in these two cases, and to the persistence of the instability waves over a longer or shorter distance that they induce. This could be explored in the future.

\section{Appendix}

In order to further illustrate the effects of the nozzle-exit mean velocity profile, two jets with untripped boundary layers, denoted as Turb0\% and Lam0\%, are considered in this appendix. As previous jets, they are isothermal, and have Mach and Reynolds numbers equal to $\mathrm{M}=0.9$ and $\mathrm{Re}_{D}=5 \times 10^{4}$. Their velocity profiles at the pipe-nozzle inlet are the transitional and laminar boundary-layer profiles used for Turb6\% and Lam6\%, respectively. Given the absence of flow forcing in the nozzle, the turbulence intensities at the exit are very close to $0 \%$.

Vorticity and pressure fields obtained for the two jets are represented in figures 15 (a,b). In both cases, the shear layers initially roll up, and vortices are then created. These vortices are smaller for Turb0\% than for Lam0\%. In addition, downstream of the first vortex pairings, the size of the turbulent structures increase abruptly in the first case, but gradually in the second one. As discussed in the paper, these results are due to the better coupling, in term of frequency, of the near-nozzle instability waves with the downstream shear-layer instability waves for a laminar boundary-layer profile than for a non-laminar one. In the pressure fields, very strong acoustic waves are generated by the pairings and mutual interactions of the vortices in the mixing layers for the two jets, as expected for untripped jets [2, 24].

The variations of the rms axial velocity fluctuations at $r=r_{0}$ are shown in figure 16(a). For both Turb0\% and Lam0\%, a hump is observed around the position of the first stage of vortex pairings in the mixing layers. However, the turbulence levels reach lower maximum values and decrease more rapidly after the peak for Turb0\% than for Lam0\%. The pressure spectra obtained at $150 r_{0}$ from the nozzle for $\phi=90^{\circ}$ are depicted in figure 16 b). Unsurprisingly, the noise levels are significantly higher for Turb0\% and Lam0\% with untripped boundary layers than for Turb6\% and Lam6\% with initially disturbed flow conditions [8]. Nevertheless, they are lower for Turb0\% than for Lam0\%, especially at low frequencies, and no component at the vortex-pairing frequency emerges in the first case contrary to the second one. 

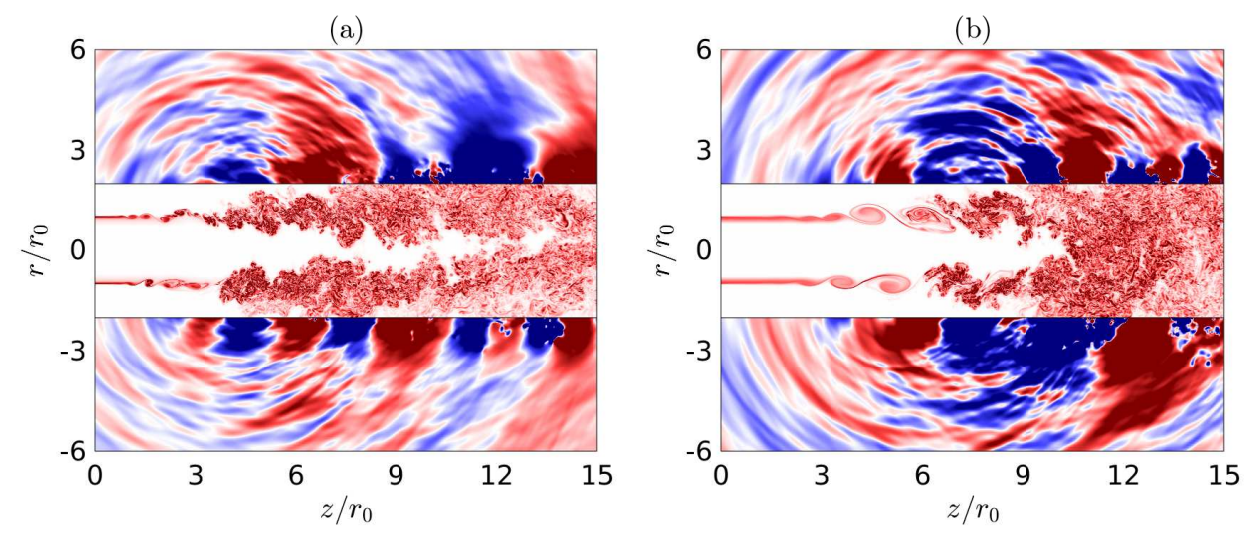

Fig. 15 Vorticity norm and pressure fluctuations for (a) Turb0\% and (b) Lam0\%. The color scales vary between $\pm 15 u_{j} / r_{0}$ and $\pm 3.6 \times 10^{-3} p_{a}$, respectively, from blue to red.
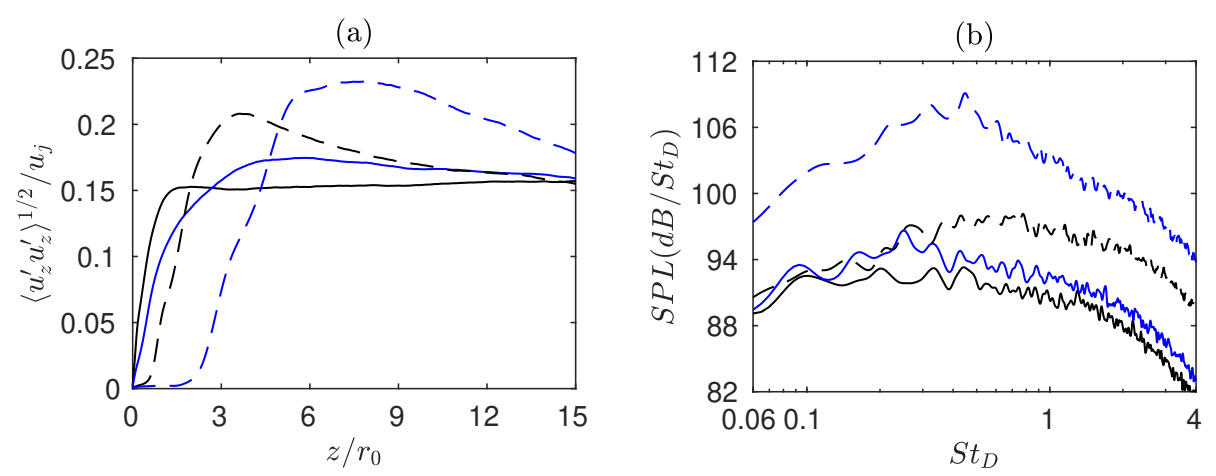

Fig. 16 Representation of (a) the rms values of axial velocity at $r=r_{0}$ and (b) far-field sound pressure levels for $\phi=90^{\circ}$ : Turb6\%, Lam6\%, - - Turb0\%, - - Lam0\%. 


\section{Acknowledgments}

This work was granted access to the HPC resources of PMCS2I (POle de Modélisation et de Calcul en Sciences de l'Ingénieur et de l'Information) of Ecole Centrale de Lyon, PSMN (Pole Scientifique de Modélisation Numérique) of ENS de Lyon and P2CHPD (Pole de Calcul Hautes Performances Dédiés) of Université Lyon I, members of FLMSN (Fédération Lyonnaise de Modélisation et Sciences Numériques), partner of EQUIPEX EQUIP@MESO, and to the resources of IDRIS (Institut du Développement et des Ressources en Informatique Scientifique) under the allocation 2019-2a0204 made by GENCI (Grand Equipement National de Calcul Intensif). It was performed within the framework of the LABEX CeLyA (ANR-10-LABX-0060) of Université de Lyon, within the program Investissements d'Avenir (ANR-16-IDEX-0005) operated by the French National Research Agency (ANR). The author gratefully acknowledges Roberto Sabatini for his help in performing linear stability analyses from the jet mean flow profiles.

\section{References}

[1] Mollo-Christensen, E., Kolpin, M. A., and Martucelli, J. R., "Experiments on jet flows and jet noise far-field spectra and directivity patterns," J. Fluid Mech., Vol. 18, No. 2, 1964, pp. 285-301. doi:10.1017/S0022112064000209.

[2] Zaman, K. B. M. Q., “Effect of initial condition on subsonic jet noise,” AIAA J., Vol. 23, No. 9, 1985, pp. 1370-1373. doi:10.2514/3.9094.

[3] Sato, H., "Experimental investigation on the transition of laminar separated layer," J. Phys. Soc. Jpn., Vol. 11, No. 6, 1956, pp. 702-709. doi:10.1143/JPSJ.11.702.

[4] Zaman, K. B. M. Q., and Hussain, A. K. M. F., “Turbulence suppression in free shear flows by controlled excitation,” J. Fluid Mech., Vol. 103, 1981, pp. 133-159. doi:10.1017/S0022112081001274.

[5] Gutmark, E., and Ho, C.-M., "Preferred modes and the spreading rates of jets," Phys. Fluids, Vol. 26, No. 10, 1983, pp. 2932-2938. doi:10.1063/1.864058.

[6] Bradshaw, P., "The effect of initial conditions on the development of a free shear layer," J. Fluid Mech., Vol. 26, No. 2, 1966, pp. 225-236. doi:10.1017/S0022112066001204.

[7] Bridges, J. E., and Hussain, A. K. M. F., "Roles of initial conditions and vortex pairing in jet noise," J. Sound Vib., Vol. 117, No. 2, 1987, pp. 289-311. doi:10.1016/0022-460X(87)90540-2.

[8] Bogey, C., Marsden, O., and Bailly, C., "Influence of initial turbulence level on the flow and sound fields of a subsonic jet at a diameter-based Reynolds number of 10," J. Fluid Mech., Vol. 701, 2012, pp. 352-385. doi:10.1017/jfm.2012.162.

[9] Raman, G., Zaman, K. B. M. Q., and Rice, E. J., "Initial turbulence effect on jet evolution with and without tonal excitation," Phys. Fluids A, Vol. 1, No. 7, 1989, pp. 1240-1248. doi:10.2514/6.1987-2725.

[10] Morris, P. J., and Zaman, K. B. M. Q., "Velocity measurements in jets with application to noise source modelling," J. Sound Vib., Vol. 329, No. 4, 2009, pp. 394-414. doi:10.1016/j.jsv.2009.09.024.

[11] Viswanathan, K., and Clark, L. T., "Effect of nozzle internal contour on jet aeroacoustics," Int. J. Aeroacoust., Vol. 3, No. 2, 2004, pp. 103-135. doi:10.1260/1475472041494819.

[12] Zaman, K. B. M. Q., "Effect of initial boundary-layer state on subsonic jet noise," AIAA J., Vol. 50, No. 8, 2012, pp. $1784-1795$. doi:10.2514/1.J051712.

[13] Karon, A. Z., and Ahuja, K. K., "Effect of nozzle-exit boundary layer on jet noise," Tech. Rep. 2013-0615, AIAA Paper, 2013. doi:10.2514/6.2013-615.

[14] Fontaine, R. A., Elliott, G. S., Austin, J. M., and Freund, J. B., "Very near-nozzle shear-layer turbulence and jet noise," J. Fluid Mech., Vol. 770, 2015, pp. 27-51. doi:10.1017/jfm.2015.119.

[15] Zaman, K. B. M. Q., "Increased jet noise due to a "nominally laminar" state of nozzle exit boundary layer," Tech. Rep. 2017-219440, NASA TM, 2017.

[16] Brès, G. A., Jordan, P., Jaunet, V., Le Rallic, M., Cavalieri, A. V. G., Towne, A., Lele, S. K., Colonius, T., and Schmidt, O. T., "Importance of the nozzle-exit boundary-layer state in subsonic turbulent jets," J. Fluid Mech., Vol. 851, 2018 , pp. 83-124. doi:10.1017/jfm.2018.476. 
[17] Bogey, C., and Sabatini, R., "Effects of nozzle-exit boundary-layer profile on the initial shear-layer instability, flow field and noise of subsonic jets," J. Fluid Mech., Vol. 876, 2019, pp. 288-325. doi:10.1017/jfm.2019.546.

[18] Bogey, C., and Marsden, O., "Simulations of initially highly disturbed jets with experiment-like exit boundary layers," AIAA J., Vol. 54, No. 4, 2016, pp. 1299-1312. doi:10.2514/1.J054426.

[19] Bogey, C., Marsden, O., and Bailly, C., "Large-Eddy Simulation of the flow and acoustic fields of a Reynolds number $10^{5}$ subsonic jet with tripped exit boundary layers," Phys. Fluids, Vol. 23, No. 3, 2011, p. 035104. doi:10.1063/1.3555634.

[20] Bogey, C., Marsden, O., and Bailly, C., "On the spectra of nozzle-exit velocity disturbances in initially nominally turbulent, transitional jets," Phys. Fluids, Vol. 23, No. 9, 2011, p. 091702. doi:10.1063/1.3642642.

[21] Bogey, C., Marsden, O., and Bailly, C., "Effects of moderate Reynolds numbers on subsonic round jets with highly disturbed nozzle-exit boundary layers," Phys. Fluids, Vol. 24, No. 10, 2012, p. 105107. doi:10.1063/1.4757667.

[22] Bogey, C., and Marsden, O., "Identification of the effects of the nozzle-exit boundary-layer thickness and its corresponding Reynolds number in initially highly disturbed subsonic jets," Phys. Fluids, Vol. 25, No. 5, 2013, p. 055106. doi:10.1063/1. 4807071 .

[23] Morris, S. C., and Foss, J. F., "Turbulent boundary layer to single-stream shear layer: the transition region," J. Fluid Mech., Vol. 494, 2003, pp. 187-221. doi:10.1017/S0022112003006049.

[24] Bogey, C., and Bailly, C., "Influence of nozzle-exit boundary-layer conditions on the flow and acoustic fields of initially laminar jets,” J. Fluid Mech., Vol. 663, 2010, pp. 507-539. doi:10.1017/S0022112010003605.

[25] Bogey, C., de Cacqueray, N., and Bailly, C., "A shock-capturing methodology based on adaptative spatial filtering for high-order non-linear computations," J. Comput. Phys., Vol. 228, No. 5, 2009, pp. 1447-1465. doi:10.1016/j.jcp.2008.10.042.

[26] Berland, J., Bogey, C., Marsden, O., and Bailly, C., "High-order, low dispersive and low dissipative explicit schemes for multiple-scale and boundary problems," J. Comput. Phys., Vol. 224, No. 2, 2007, pp. 637-662. doi:10.1016/j.jcp.2006.10.017.

[27] Bogey, C., de Cacqueray, N., and Bailly, C., "Finite differences for coarse azimuthal discretization and for reduction of effective resolution near origin of cylindrical flow equations," J. Comput. Phys., Vol. 230, No. 4, 2011, pp. 1134-1146. doi:10.1016/j.jcp.2010.10.031.

[28] Fauconnier, D., Bogey, C., and Dick, E., "On the performance of relaxation filtering for large-eddy simulation." J. Turbulence, Vol. 14, No. 1, 2013, pp. 22-49. doi:10.1080/14685248.2012.740567.

[29] Kremer, F., and Bogey, C., "Large-eddy simulation of turbulent channel flow using relaxation filtering: Resolution requirement and Reynolds number effects," Comput. Fluids, Vol. 116, 2015, pp. 17-28. doi:10.1016/j.compfluid.2015.03.026.

[30] Tam, C. K. W., and Dong, Z., "Radiation and outflow boundary conditions for direct computation of acoustic and flow disturbances in a nonuniform mean flow," J. Comput. Acous., Vol. 4, No. 2, 1996, pp. 175-201. doi:10.1142/S0218396X96000040.

[31] Bogey, C., "Grid sensitivity of flow field and noise of high-Reynolds-number jets computed by large-eddy simulation," Int. J. Aeroacoust., Vol. 17, No. 4-5, 2018, pp. 399-424. doi:10.1177/1475472X18778287.

[32] Viswanathan, K., "Distributions of noise sources in heated and cold jets: are they different?" Int. J. Aeroacoust., Vol. 9, No. 4-5, 2010, pp. 589-625. doi:10.1260/1475-472X.9.4-5.589.

[33] Michalke, A., "Survey on jet instability theory," Prog. Aerosp. Sci., Vol. 21, 1984, pp. 159-199. doi:10.1016/0376-0421(84) 90005-8.

[34] Morris, P. J., “The instability of high speed jets," Int. J. Aeroacoust., Vol. 9, No. 1-2, 2010, pp. 1-50. doi:10.1260/1475472X.9.1-2.1.

[35] Hussain, A. K. M. F., “Coherent structures and turbulence,” J. Fluid Mech., Vol. 173, 1986, pp. 303-356. doi:10.1017/ S0022112086001192.

[36] Fieldler, H. E., “Coherent structures in turbulent flows," Prog. Aerosp. Sci., Vol. 25, 1988, pp. 231-269. doi:10.1016/03760421(88)90001-2.

[37] Brown, G. L., and Roshko, A., "On density effects and large structure in turbulent mixing layers," J. Fluid Mech., Vol. 64, No. 4, 1974, pp. 775-816. doi:10.1017/S002211207400190X. 
[38] Arndt, R. E. A., Long, D. F., and Glauser, M. N., "The proper orthogonal decomposition of pressure fluctuations surrounding a turbulent jet," J. Fluid Mech., Vol. 340, 1997, pp. 1-33. doi:10.1017/S0022112097005089.

[39] Morris, P. J., "Viscous stability of compressible axisymmetric jets," AIAA J., Vol. 21, No. 4, 1983, pp. 481-482. doi: $10.2514 / 3.8101$.

[40] Freymuth, P., "On transition in a separated laminar boundary layer," J. Fluid Mech., Vol. 25, No. 4, 1966 , pp. 683-704. doi:10.1017/S002211206600034X.

[41] Suzuki, T., and Colonius, T., "Instability waves in a subsonic round jet detected using a near-field phased microphone array," J. Fluid Mech., Vol. 565, 2006, pp. 197-226. doi:10.1017/S0022112006001613.

[42] Bell, J. H., and Mehta, R. D., "Development of a two-stream mixing layer from tripped and untripped boundary layers," AIAA J., Vol. 28, No. 12, 1990, pp. 2034-2042. doi:10.2514/3.10519.

[43] Morris, P. J., “The spatial viscous instability of axisymmetric jets,” J. Fluid Mech., Vol. 77, No. 3, 1976, pp. $511-529$. doi:10.1017/S0022112076002231.

[44] Winant, C., and Browand, F., "Vortex pairing : the mechanism of turbulent mixing-layer growth at moderate Reynolds number," J. Fluid Mech., Vol. 63, No. 2, 1974, pp. 237-255. doi:10.1017/S0022112074001121.

[45] Zaman, K. B. M. Q., "Flow field and near and far sound field of a subsonic jet," J. Sound Vib., Vol. 106, No. 1, 1986, pp. 1-16. doi:10.1016/S0022-460X(86)80170-5.

[46] Tam, C. K. W., “Jet noise: Since 1952,” Theor. Comput. Fluid Dyn., Vol. 10, No. 1-4, 1998, pp. 393-405. doi:10.1007/ s001620050072.

[47] Chu, W. T., and Kaplan, R. E., "Use of a spherical concave reflector for jet-noise-source distribution diagnosis," J. Acoust. Soc. Am., Vol. 59, No. 6, 1976, pp. 1268-1277. doi:10.1121/1.381014.

[48] Fisher, M. J., Harper-Bourne, M., and Glegg, S. A. L., "Jet engine noise source location: The polar correlation technique," J. Sound Vib., Vol. 51, No. 1, 1977, pp. 23-54. doi:10.1016/S0022-460X(77)80111-9.

[49] Lee, S. S., and Bridges, J., "Phased-array measurements of single flow hot jets," Tech. Rep. 2005-213826, NACA TM, 2005.

[50] Kibens, V., "Discrete noise spectrum generated by acoustically excited jet," AIAA J., Vol. 18, No. 4, 1980 , pp. $434-441$. doi: $10.2514 / 3.50776$.

[51] Zaman, K. B. M. Q., and Hussain, A. K. M. F., “Turbulence suppression in free shear flows by controlled excitation,” J. Fluid Mech., Vol. 103, 1981, pp. 133-159. doi:10.1017/S0022112081001274.

[52] Bechert, D., and Pfizenmaier, E., "On the amplification of broad band jet noise by a pure tone excitation," J. Sound Vib., Vol. 43, No. 3, 1975, pp. 581-587. doi:10.1016/0022-460X(75)90015-2.

[53] Crighton, D. G., "Acoustics as a branch of fluid mechanics,” J. Fluid Mech., Vol. 106, 1981, pp. 261-298. doi:10.1017/ S0022112081001602.

[54] Zaman, K. B. M. Q., "Far-field noise of a subsonic jet under controlled excitation,” J. Fluid Mech., Vol. 152, 1985 , pp. 83-111. doi:10.1017/S0022112085000581. 